\title{
Estimating the Production and Release of Ascospores from a Field-Scale Source of Fusarium graminearum Inoculum
}

Aaron J. Prussin, II, Nicole A. Szanyi, and Patricia I. Welling, Department of Plant Pathology, Physiology, and Weed Science, Virginia Tech, Blacksburg, VA 24061-0390; Shane D. Ross, Department of Engineering Science and Mechanics, Virginia Tech, Blacksburg, VA 24061-0219; and David G. Schmale, III, Department of Plant Pathology, Physiology, and Weed Science, Virginia Tech, Blacksburg, VA 24061-0390

\begin{abstract}
Prussin, A. J., II, Szanyi, N. A., Welling, P. I., Ross, S. D., and Schmale, D. G., III. 2014. Estimating the production and release of ascospores from a field-scale source of Fusarium graminearum inoculum. Plant Dis. 98:497-503.

Fusarium head blight (FHB) is a devastating disease of wheat and barley caused by the fungus Fusarium graminearum. The fungus produces spores that may be transported over long distances in the atmosphere. In order to predict the atmospheric transport of $F$. graminearum, the production and release of ascospores must be known. We conducted a series of laboratory and field experiments to estimate perithecia production and ascospore release from a field-scale source of $F$. graminearum inoculum. Perithecia were generated on artificial (carrot agar) and natural (corn stalk) substrates. Artificial substrates produced $15 \pm 0.4$ perithecia $/ \mathrm{cm}^{2}$, and natural substrates produced $44 \pm 2$ peri-

thecia/ $\mathrm{cm}^{2}$. Eighty perithecia were excised from both substrate types and allowed to release ascospores every $24 \mathrm{~h}$. Perithecia generated from artificial and natural substrates released a mean of $104 \pm 5$ and $276 \pm$ 16 ascospores over 10 days, respectively. A volumetric spore trap was placed inside a 1-acre clonal source of inoculum in 2011 and 2012. Results indicated that ascospores were released predominantly during the night (1900 to 0700). Estimates of ascospore production for our field-scale sources of inoculum were approximately 400 million ascospores/day for 10 days. Mathematical models can use estimates of ascospore production to assist in predicting the transport of $F$. graminearum.
\end{abstract}

Fusarium graminearum, causal agent of Fusarium head blight (FHB) in wheat and barley, is another important fungal plant pathogen that is transported through the atmosphere $(24,29,32)$. This pathogen of economic concern has caused over $\$ 3$ billion in crop losses over the past two decades in the United States $(19,22,26)$, in large part due to the production of deoxynivalenol, a mycotoxin that contaminates grain and renders it unfit for consumption $(31,32)$. Liberation, horizontal transport, and deposition are the aerobiological processes that govern atmospheric transport of plant pathogens such as F. graminearum (13).

Ascospores of $F$. graminearum are liberated from fruiting bodies known as perithecia. Warm temperatures and water availability promote the development of mature perithecia (10). Mature perithecia produce multiple asci, and each ascus contains eight ascospores (34). Ascospore release from mature perithecia is triggered in part by an increase in relative humidity $(32,35)$. Ascospores are typically released distances of about $5 \mathrm{~mm}$, at a speed of $34.5 \mathrm{~m} / \mathrm{s}$, and acceleration of $860,000 \mathrm{~g}(25,34)$. There have been conflicting reports documenting the timing of ascospore release. Some studies have shown that ascospores are released predominantly at night due to an increase in relative humidity $(11,21,36)$. However, studies by Maldonado-Ramirez (17) suggested that ascospores are released primarily during daylight hours. Viable spores of $F$. graminearum have been recovered from the lower atmosphere during all times of the day and night $(18,24,29)$. Schmale et al. $(27,28)$ reported that spores of $F$. graminearum were deposited predominantly during the night, suggesting that deposition rates might be higher at night due to an inversion layer being formed from a rapid cooling of earth's surface compared to the atmosphere (20).

FHB is managed in part by the use of risk assessment models that guide the appropriate timing of fungicide application $(7,8)$.

Corresponding author: David G. Schmale, III, E-mail: dschmale@vt.edu

Accepted for publication 25 October 2013.

http://dx.doi.org/10.1094/PDIS-04-13-0404-RE

(C) 2014 The American Phytopathological Society
These models leverage knowledge of environmental factors and disease reports. However, they fail to address the atmospheric transport of $F$. graminearum spores from potential inoculum sources.

Aylor et al. (1-4) pioneered the development of mathematical models to predict the atmospheric movement of plant-pathogenic fungi. The accuracy and reliability of these models is tightly linked to uncertainties regarding spore release dynamics and the potential source strength of the inoculum source $\left(Q_{0}\right)$, which depends on spore production and escape (1). Differences in spore production and release dynamics can affect the magnitude of the dispersal kernels for fungal spores 100 to 1,000 times (1). Consequently, there is a need to develop a reliable estimate of spore production to increase the accuracy and precision of these models.

The specific objectives of this study were to: (i) measure the production of perithecia and the release of ascospores from natural and artificial substrates, (ii) monitor the patterns of ascospore release from a field-scale source of inoculum, and (iii) develop an accurate estimate of ascospore production for a field-scale source of inoculum of $F$. graminearum. We hypothesized that (i) perithecia production and ascospore release of $F$. graminearum vary between natural and artificial substrates and (ii) ascospores of $F$. $g r a-$ minearum are released under field conditions primarily during the night. To test these hypotheses, a series of laboratory and field experiments were conducted. Perithecia were generated on artificial (carrot agar) and natural (corn stalk) substrates, and individual perithecia were excised from both substrate types and allowed to release ascospores every $24 \mathrm{~h}$. A volumetric spore trap was placed inside a 1-acre clonal source of inoculum in 2011 and 2012. Results from these experiments were used to estimate ascospore production for a field-scale source of inoculum of $F$. graminearum.

\section{Materials and Methods}

Field experiments. Field studies were conducted at Virginia Tech's Kentland Farm in Blacksburg, VA during the spring of 2011 and 2012. The Kentland Farm is composed of about 810 hectares of farmland. Two hectares of winter wheat (untreated Southern States variety SS560) were planted in October 2010 for the 2011 
field campaign and October 2011 for the 2012 field campaign. The winter wheat field was not treated with any fungicides.

Inoculum was prepared as described previously (23). Briefly, 50 18.92-liter buckets were filled with approximately $15 \mathrm{~cm}$ of cut, dried, and autoclaved corn stalk pieces. The corn stalks were inoculated with Fg_Va_GPS13N4_3ADON (hereafter referred to as FGVA4), and the fungus was allowed to colonize the corn stalks for approximately 10 weeks at room temperature.

A plot area of $3,716 \mathrm{~m}^{2}(0.372 \mathrm{ha})$ of wheat was subdivided into 100 square plots ( 10 rows of 10 plots, $6.1 \times 6.1 \mathrm{~m}$ ). Field inoculations were performed on 2 May 2011 and 16 April 2012 by placing corn stalks from each of the 50 buckets into 50 of the subplots in a checkerboard pattern (stalks from one bucket were used for each of the subplots).

Production of perithecia on different substrates. Perithecia were generated on artificial (carrot agar, CA) and natural (corn stalk) substrates $(15,25,33,34)$. Cultures of FGVA4 and Fg_Va_N10_15ADON (hereafter referred to as FGVA10) were grown on petri dishes $\left(19.63 \mathrm{~cm}^{2}\right)$ containing CA, with fresh organic carrot concentrations ranging from 0 to $400 \mathrm{~g}$ carrots/liter media. Cultures for both FGVA4 and FGVA10 were grown at 0, 100, 200, 225, 250, 275, 300, and 400 g carrots/liter media. Eight dishes at each concentration were inoculated with FGVA4 and FGVA10. The total sample size was 128 dishes; 64 dishes were inoculated with FGVA4 and 64 dishes were inoculated with FGVA10. Preliminary experiments (data not shown) indicated the largest differences in the number of perithecia formed were between 200 and $300 \mathrm{~g}$ carrots/liter media, which suggested that we should increase our data collection in this range. The cultures were incubated at room temperature until aerial mycelium covered the petri dishes (approximately 5 to 7 days). A 2.5\% Tween 60 (Product No. 278622500; Acros Organics, Fair Lawn, NJ) solution was then added to the cultures and mycelium was flattened using a sterile rod. The cultures were put under a 12-12 h light-dark cycle to encourage perithecia development. Perithecia were observed approximately 3 days after the addition of the $2.5 \%$ Tween 60 solution. The total number of perithecia formed on each culture plate was quantified 9 days after the addition of the Tween solution. The average number of perithecia/ $\mathrm{cm}^{2}$ on the CA surface was calculated for both FGVA4 and FGVA10 isolates for all eight nutrient concentrations.

Perithecia were also quantified from the inoculated corn stalks incubated in the field under natural conditions. Three corn stalks from 49 of the 50 (samples from one subplot were lost) inoculated subplots in 2012 were randomly collected (17 May), giving a total sample size of 147 corn stalks. The corn stalks were brought back to the lab, and the total number of perithecia in six random 0.25 $\mathrm{cm}^{2}$ sections were counted. Six $0.25 \mathrm{~cm}^{2}$ rectangles were painted onto mesh window screening containing a screen size of $2.25 \mathrm{~mm}^{2}$. The window screening was then wrapped around the corn stalks, allowing for the unbiased selection of the six $0.25 \mathrm{~cm}^{2}$ areas to count.

Quantification of ascospores from perithecia generated on artificial and natural substrates. Ascospores were quantified from perithecia generated on artificial (carrot agar, CA) and natural (corn stalk) substrates. For perithecia from artificial substrates, cultures of FGVA4 were grown on sterile circles of filter paper $(0.25 \mathrm{~cm}$ in diameter) scattered on plates of CA containing $400 \mathrm{~g}$ carrots/liter media. Perithecia production was encouraged using a $2.5 \%$ Tween 60 solution as described above. The cultures were checked every day for mature perithecia, characterized as being spherical bodies, bluish-black in color (26). As soon as mature perithecia appeared, as early as 3 days, 80 pieces of filter paper each containing an individual perithecium were transferred and placed on the lids of 200- $\mu$ l microcentrifuge tubes (Product No. 1402-1880; USA Scientific, Ocala, FL), with the ostiole of the perithecium facing down. The moisture on the filter paper from the CA held the filter paper containing the perithecia in place on the lid. These discharge chambers, containing filter paper with a single perithecium attached to the lid, were left at room temperature to allow the perithecia to release ascospores into the tubes for $24 \mathrm{~h}$. After $24 \mathrm{~h}$, each lid was transferred to a new discharge chamber. The chambers that had been exposed to ascospore discharge from the perithecia were centrifuged at 2,000 $\times g$ for approximately $5 \mathrm{~s}$, and the ascospores were resuspended in $12 \mu \mathrm{l}$ of sterile water and quantified using a hemacytometer (Product No. 3100; Hausser Scientific, Horsham, PA) and light microscope. Ascospores were quantified from perithecia for a total of 10 discharge days.

For perithecia from natural substrates, corn stalks inoculated with FGVA4 were collected 8 days after they were placed into the field, corresponding to the first day perithecia appeared. Sixty perithecia were removed from the corn stalks by using a scalpel to cut off portions of corn stalk containing single perithecium and placing them in discharge chambers by using superglue to affix the corn stalk to the PCR tube lid with the ostiole of the perithecium facing down. Ascospores were quantified from perithecia for a total of 10 discharge days.

Since each mature ascus should contain eight ascospores (33), the number of asci in a perithecium was calculated by dividing the total number of ascospores by eight.

Timing of ascospore release from a field-scale source of inoculum under field conditions. A volumetric spore sampler (Quest Developments, Brits, South Africa) was placed in the center of a 1acre wheat field artificially inoculated with FGVA4 colonized corn stalks in 2011 and 2012, as described above (23). The sampling orifice of the volumetric spore sampler was at approximately half the height of the wheat canopy $(\sim 0.5 \mathrm{~m})$, and a $6.1 \times 6.1 \mathrm{~m}$ area of wheat was removed in the subplot where the volumetric spore sampler was placed to allow it to move freely. The sampling orifice of the volumetric spore sampler was $0.5 \mathrm{~m}$ above the inoculum source. The sampler collected a continuous sample of ascospores on a rotating disc coated with silicone grease. The flow rate of the volumetric spore sampler was measured three independent times using an MMA-5 flowmeter (Dwyer Instruments Inc., Michigan City, IN) and was 7.8 standard cubic feet per hour for all three measurements (two measurements were taken in August 2009 and one measurement was taken in May 2011). This flow rate was used to compute the concentration of ascospores at the volumetric spore sampler for each time point in 2011 and 2012.

Samples were collected from 1700 on 19 May to 0800 on 3 June 2011 and 1800 on 26 April to 1100 on 14 May 2012, corresponding with when inoculum was present. To assess the background concentration of ascospores, samples were collected with the Quest sampler between 1700 on 9 April and 0400 on 16 April 2012, corresponding to the week before inoculum was placed in the field. Samples collected by the Quest sampler were brought back to the lab and stained with Calberla's Stain (Multidata Inc., Saint Louis Park, MN) to allow the $F$. graminearum ascospores to be visualized. Ascospores of F. graminearum were identified based on morphological characteristics of spores containing 3 to 4 septa and having rounded edges, as previously described (26). The total number of $F$. graminearum ascospores captured by the Quest sampler was quantified hourly for each period of sampling in both years. Due to the large sample size and very high number of ascospores being released from our inoculated source, a method had to be developed to allow for the efficient collection of data. The total number of ascospores in one field of view at $\times 400$ was quantified at the two edges of each hourly interval (i.e., 0600 to 0615 and 0645 to 0700 ). The average of the two counts was taken and extrapolated to get an estimate of the total number of ascospores released during the $1-\mathrm{h}$ period. To validate this counting method, six time points were chosen where all of the ascospores were counted across the entire 1-h zone and compared to the counting method described above. Two time points at random were chosen for high, medium, and low counts which corresponded to less than 10 , between 10 and 40, and greater than 40 ascospores in one field of view at $\times 400$, respectively. The correlation coefficient for the association between the different counting methods (counts of sections to estimate hourly counts and total counts of hourly intervals) was 0.97 . 
Estimation of ascospore production of a field-scale source of inoculum. Ascospore production was estimated using a natural (corn stalks) substrate. The total number of corn stalks (50 buckets) placed across our $\sim 1$-acre source was estimated from counts of corn stalks in six of the buckets. The aboveground surface area of these stalks (the area not in contact with the soil surface) was calculated by subtracting the surface area of the corn stalk in contact with the ground (perithecia forming from this surface would not be able to contribute to ascospores in the atmosphere) from total corn stalk surface area, which was calculated from the measured corn stalk radius and length and the assumption that the corn stalk was a perfect cylinder. We only considered the area density of perithecia projected onto the surface of a cylinder approximately matching the radius and height of each corn stalk. The detailed folded geometry of a corn stalk was not considered. The surface area of the corn stalk in contact with the ground was calculated by laying 147 corn stalks in a bed of flour. Each stalk was removed from the flour, and the surface area covered in flour was calculated and subtracted from the total surface of the stalk. Ascospore production was then estimated using the following equation:

Ascospore production $=[($ number of 18.92-liter buckets released $) *($ number of corn stalks/18.92-liter bucket)*(exposed surface area/corn stalk)* (number of perithecia/surface area)*(number of ascospores/perithecium)]/total sampling time (days)

The resulting number gives an estimate of the total number of ascospores potentially released from a field-scale source of inoculum containing 5,800 stalks. It is important to note that this estimate does not consider the fraction of spores that escape the crop canopy as included in other estimates, such as $Q_{0}(1,4)$.

Statistical analyses. Analysis of variance (ANOVA) was used to test for significant differences in number of perithecia formed for different agar concentrations ( 0 to $400 \mathrm{~g}$ carrots/liter media) between two $F$. graminearum isolates (FGVA4 and FGVA10), and the potential interaction between isolates and media. ANOVA was used to determine significant differences in ascospore release between day (0700 to 1900) and night (1900 to 0700), in 2011 and 2012. Data were reported for hourly release for 14 consecutive days in 2011 and 18 consecutive days in 2012, and ANOVA was used to determine significant differences in the number of ascospores released between each hour of the day. Finally, ANOVA was used to test for plot uniformity across the 1-acre inoculated plot. When controlling for the location in the inoculated field a corn stalk came

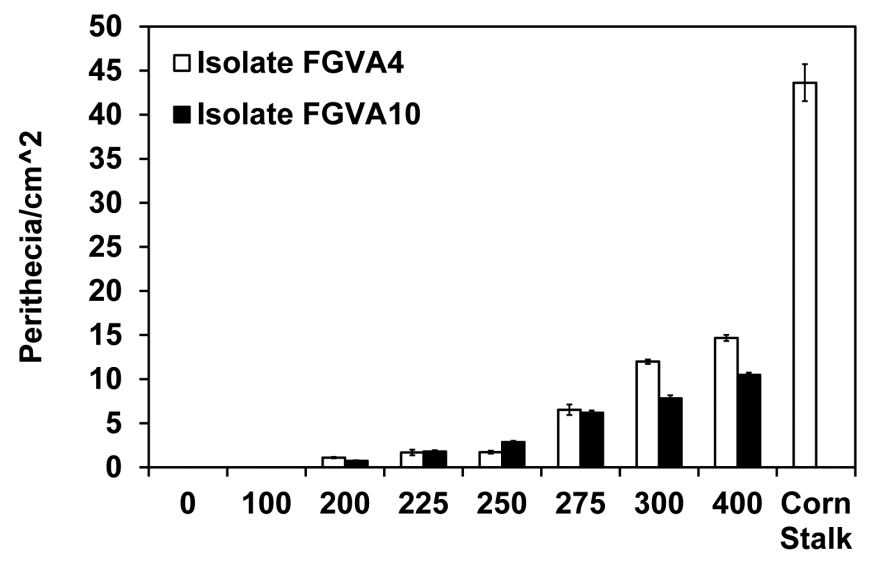

\section{Carrot agar (g/L) or corn stalk}

Fig. 1. Production of perithecia on artificial (carrot agar, $\mathrm{CA}$ ) and natural (corn stalk) substrates. CA contained different amounts of carrots ranging from $0 \mathrm{~g}$ carrots/liter media to $400 \mathrm{~g}$ carrots/liter media. CA plates were inoculated with two different Fusarium graminearum isolates naturally recovered in Virginia, FGVA4 and FGVA10. A sample size of eight was used for each isolate at each CA concentration. Corn stalks inoculated with FGVA4 were recovered from a 1-acre field-scale source and the number of perithecia/ $\mathrm{cm}^{2}$ was quantified $(n=147)$. Error bars represent variation among replicates in the number of perithecia produced. from, we tested for significant differences in total surface area, surface area in the ground, and number of perithecia $/ \mathrm{cm}^{2}$ formed. Statistical analyses were performed using JMP System for Windows (Release 10, SAS Institute Inc., Cary, NC). For all of the tests, significance was evaluated at $P<0.05$.

\section{Results}

Production of perithecia on different substrates. For the production of perithecia on $\mathrm{CA}$, an increasing number of perithe$\mathrm{cia} / \mathrm{cm}^{2}$ were observed as the concentration of carrots increased from 0 to $400 \mathrm{~g}$ (Fig. 1). No perithecia appeared for either FGVA4 or FGVA10 isolates on plates with nutrient concentrations of $0 \mathrm{~g}$ carrots/liter media or $100 \mathrm{~g}$ carrots/liter media. The differences in the number of perithecia/ $/ \mathrm{cm}^{2}$ as the concentration of carrots increased was significant for both FGVA4 $(P<0.001)$ and FGVA10 $(P<0.001)$. CA that contained $400 \mathrm{~g}$ of carrots per liter produced an average of $14.7 \pm 0.36$ perithecia/ $\mathrm{cm}^{2}$ for FGVA4 and $10.5 \pm$ 0.24 perithecia $/ \mathrm{cm}^{2}$ for FGVA10. There was no significant difference in the number of perithecia/ $\mathrm{cm}^{2}$ formed $(P=0.243)$ between the two isolates studied (FGVA4 and FGVA10). The interaction between isolate and media (amount of carrots) was significant $(P<$ 0.001).

Corn stalks inoculated with FGVA4 produced an average of $44 \pm$ 2.1 perithecia/ $/ \mathrm{cm}^{2}$ (Fig. 1). Approximately three times more perithecia appeared on corn stalks per unit area than when the fungus was grown on CA at a concentration of $400 \mathrm{~g}$ carrots/liter media.

Quantification of ascospores from perithecia generated on artificial and natural substrates. The total number of ascospores and length of time ascospores were released were quantified from perithecia generated on CA (400 g carrots/liter media) and corn stalks. Over a 10-day period, immediately following mature perithecia development, a mean of $104 \pm 5$ ascospores and $276 \pm 16$ ascospores were released from perithecia generated on CA $(n=71)$ and corn stalks $(n=40)$, respectively (Fig. 2, Table 1). The total number of ascospores contained within perithecia from CA and those from corn stalks were significantly different $(P<0.001)$. The number of asci contained within each perithecium was estimated to be a mean of $13 \pm 0.6$ and $34 \pm 2$ for perithecia from CA and corn stalks, respectively (Table 1 ).

Trends in daily ascospore release were also examined for the perithecia generated on the two different types of substrates (CA and corn stalks). A similar trend was observed for perithecia from both types of substrates; the number of ascospores released increased for approximately 4 or 5 days before decreasing over several days until ascospores were no longer released (Fig. 2, Table 1). The average

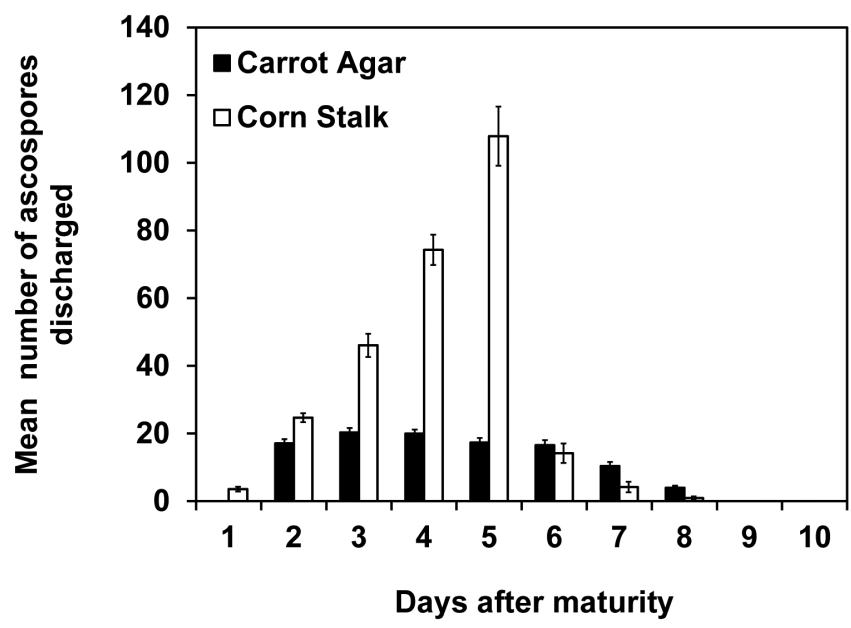

Fig. 2. The mean daily number of ascospores released from perithecia generated from carrot agar (CA) and corn stalks. CA and corn stalks were both inoculated with FGVA4 and checked daily for the formation of mature perithecia. As soon as mature perithecia were present, perithecia were extracted from the substrates and the number of ascospores released each day was studied. Sample sizes of 71 and 40 perithecia were used for $\mathrm{CA}$ and corn stalks, respectively. 
duration of the active period of ascospore release from perithecia was approximately 7 and 6 days after mature perithecia first developed on CA and corn stalks, respectively (Fig. 2, Table 1).

Timing of ascospore release from a field-scale source of inoculum under field conditions. The timing of ascospore release was studied hourly between 1700 hours 19 May to 0800 hours 3 June 2011 and 1800 hours 26 April to 1100 hours 14 May 2012 for an artificially inoculated field with FGVA4 corn stalks (Fig. 3). The majority, $98 \%(616,594 / 627,165)$ and $92 \%(264,418 / 288,508)$, of the ascospores were captured by the volumetric spore sampler between 1900 and 0700 in 2011 and 2012, respectively. In 2011, the majority of ascospores were released between 0100 and 0200 , $28 \%(175,637 / 627,165)$ of ascospores were released during this hour. In 2012, the majority of ascospores were released between 2200 and 2300, 17\% (48,180/288,508) of ascospores were released during this hour. Ascospore release was significantly different between day (0700 to 1900) and night (1900 to 0700) for 2011 ( $P$ $=0.035)$ and $2012(P=0.003)$. Additionally, the number of ascospores released from the inoculated field varied significantly among hours of the day or night in both $2011(P=0.003)$ and 2012 $(P<0.001)$. Ascospore release varied among sampling days (Figs. 4 and 5). Four and seven peak ascospore release nights (1900 to 0700) were observed in 2011 and 2012, respectively. A peak release night was assigned if more ascospores were released than the average number of ascospores released during the night (1900 to 0700) during the entire sampling period in 2011 (average number of spores released each night was 41,106) and 2012 (average number of spores released each night was 14,690). Ascospore release was significantly different across sampling days in both $2011(P<$ $0.001)$ and $2012(P<0.001)$.

In 2012, the timing of ascospore release was measured before inoculum was placed in the field. The effect of time of day on the number of ascospores released was not significant $(P=0.958)$, suggesting that the majority of ascospores being collected when inoculum was present in the field were coming from the inoculated field, rather than a background source(s). Finally, the average hourly rate (day and night combined) of ascospore release was 17.4 times greater after inoculum was placed in the field.

Estimation of ascospore production of a field-scale source of inoculum. To estimate the ascospore production of our field-scale source of inoculum, 147 corn stalks were randomly collected across the field and further analyzed in the lab. The average number of corn stalks contained within each 5-gal. bucket placed in the inoculated field was $116 \pm 7$ corn stalks. The average total surface area of the corn stalks used to inoculate the 1-acre plot was $73.4 \pm$ $2.2 \mathrm{~cm}^{2}$; however, since $20.2 \pm 0.75 \mathrm{~cm}^{2}$ was estimated to be the average surface area of the corn stalk exposed to the ground (and thus not able to contribute to the release of ascospores into the atmosphere), the total surface area of the corn stalk exposed to the atmosphere was calculated to be an average of $53.2 \pm 1.8 \mathrm{~cm}^{2}$.

Table 1. Differences in ascospore release of a single strain of Fusarium graminearum (FGVA4) from perithecia generated on artificial (carrot agar) and natural (corn stalks) substrates ${ }^{\mathrm{a}}$

\begin{tabular}{lcc}
\hline & Carrot agar $^{\mathbf{b}}$ & Corn stalks $^{\mathbf{c}}$ \\
\hline Perithecia/cm & \\
Total number of ascospores & $14.7 \pm 0.4$ & $43.6 \pm 2.1$ \\
$\quad$ released/perithecia & $104.5 \pm 5.1$ & $275.7 \pm 16.1$ \\
Total number asci/perithecium $^{\mathrm{d}}$ & $13.1 \pm 0.6$ & $34.5 \pm 2.0$ \\
Duration of ascospore release $^{\mathrm{e}}$ & $7.0 \pm 0.2$ & $5.9 \pm 0.2$ \\
Peak ascospore release day $^{\mathrm{f}}$ & $4.2 \pm 0.2$ & $4.8 \pm 0.2$ \\
\hline
\end{tabular}

${ }^{a}$ Results presented are means plus/minus standard errors.

${ }^{\mathrm{b}}$ Carrot agar concentration was $400 \mathrm{~g}$ carrots/liter media. Sample size of $\mathrm{n}=71$.

${ }^{c}$ Sample size of $\mathrm{n}=40$.

${ }^{\mathrm{d}}$ Estimated by dividing total number of ascospores by eight.

${ }^{e}$ Number of days the perithecia released ascospores after formation of mature perithecia.

${ }^{\mathrm{f}}$ Number of days after formation of mature perithecia that ascospore release was the highest.
Using equation 1 , the number of ascospores released from the inoculated field was estimated to be $3.7 \times 10^{8}$ ascospores/day based on estimates from corn stalks (Table 2).

There were no significant differences between the subplot the corn stalk came from and the number of perithecia/ $/ \mathrm{cm}^{2}(P=$ $0.417)$, total surface area $(P=0.167)$, or exposed surface area $(P=$ 0.151 ), indicating a uniform source of potential inoculum.

\section{Discussion}

Differences in ascospore production have the potential to affect the magnitude of the dispersal kernels for fungal spores (1-4). Consequently, there is a need to develop a reliable estimate of ascospore production to increase the accuracy and precision of these models. In this study, we estimated ascospore production from a 1-acre field-scale source of inoculum of a clonal isolate of F. graminearum released at Virginia Tech's Kentland Farm in Blacksburg, VA. Estimates of ascospore production for our fieldscale sources of inoculum were approximately 400 million ascospores/day over 10 days. It is important to note that our estimate does not consider the fraction of spores that escape the crop canopy as included in other estimates, such as $Q_{0}(1,4)$. This work may assist extension agents in estimating spore production, provided a reasonable estimate of ground cover of corn stalks containing mature perithecia exists. Mathematical models can use these estimates in the future to assist in predicting the long-distance transport of $F$. graminearum.
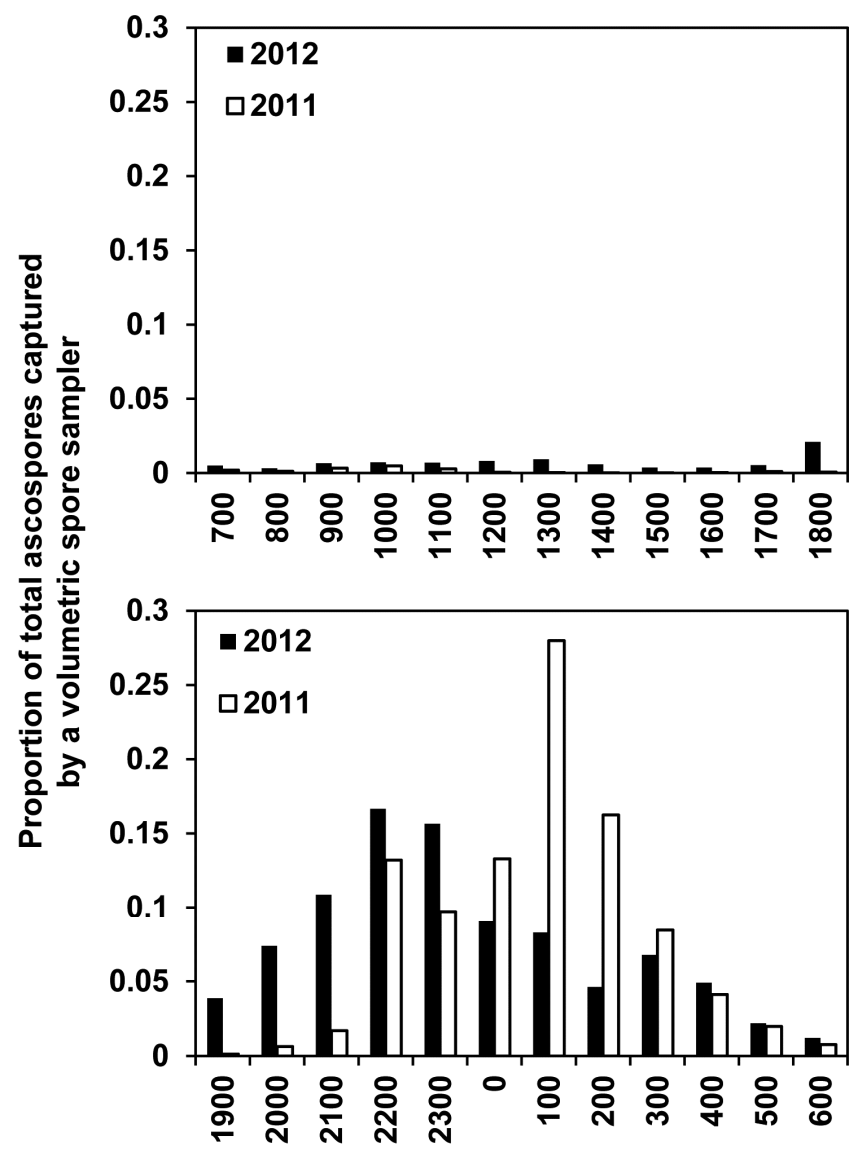

Time of day

Fig. 3. Proportion of total number of ascospores released and captured by a Quest volumetric spore sampler placed in the center of a 1-acre wheat field inoculated with FGVA4, during the day (0700-1900; top) and night (1900-0700; bottom). The volumetric spore sampler was continually sampling between 1700 hours 19 May to 0800 hours 3 June 2011 and 1800 hours 26 April to 1100 hours 14 May 2012. These dates correspond to when corn stalks inoculated with FGVA4 were present in the 1-acre wheat field. For reference, a proportion of 0.1 is equivalent to 63,000 ascospores in 2011 and 29,000 ascospores in 2012. 
Artificial substrates produced $15 \pm 0.4$ perithecia $/ \mathrm{cm}^{2}$ and natural substrates produced $44 \pm 2$ perithecia/ $/ \mathrm{cm}^{2}$. Multiple studies have reported the effect of temperature and relative humidity on perithecia development $(6,9,10,14,32)$, yet the production of perithecia under different nutrient regimes requires further attention. Hall (12) examined the effect of carbon:nitrogen ratios on the production of perithecia of Sordaria fimicola (Roberge) Ces. \& deNot, and found there was an optimal ratio to maximize perithecia development. Corn stalks have a much higher carbon: nitrogen ratio $(60: 1)$ than carrots $(27: 1)(16,30)$. The difference in the carbon:nitrogen ratios between corn stalks and carrots could explain the large difference in number of perithecia formed between corn stalks and carrot agar, at least in part. Future work might examine the effect of carbon:nitrogen ratios on perithecia development for $F$. graminearum. The large difference seen between a natural substrate (corn stalks) and an artificial substrate (carrot agar) suggests that researchers must use caution when translating laboratory measurements to the field.

Perithecia generated from artificial and natural substrates released a mean of $104 \pm 5$ and $276 \pm 16$ ascospores, respectively. A possible explanation for the difference in number of ascospores between these substrates could also be due to the carbon:nitrogen ratios in the different substrates as discussed earlier. It is possible perithecia from carrot agar might not be able to fully develop the same number of mature asci as perithecia from corn stalks (5). It is important to note that we are only able to report the number of released ascospores from each perithecium. It is possible there are more ascospores that are not released from the perithecia. Previous research suggested that if conditions for ascospore release are not optimal, ascospores get released from a perithecium as a cirrhus, due to the accumulation of mannitol $(34,35)$. Thus, we are probably underestimating the true number of ascospores contained in a perithecium and the number of asci contained within a perithecium, as our calculation was based on the number of released ascospores and knowing that each mature asci contains eight ascospores (33). Due to the differences observed in both the number of perithecia formed and number of ascospores released when two different substrates were used (carrot agar and corn stalks), it is also possible that ascospore release dynamics could be different for perithecia from natural substrates, such as corn stalks, than for perithecia from artificial substrates, such as carrot agar $(25,34)$. This is beyond the scope of this work, but should be further investigated.

Ascospores of $F$. graminearum were released under field conditions predominantly during the night (1900 to 0700). These results are consistent with previous studies $(11,21,36)$; however, they contradict one previous study (17). There is evidence that ascospores are released due to a drying-wetting cycle of perithecia, where the
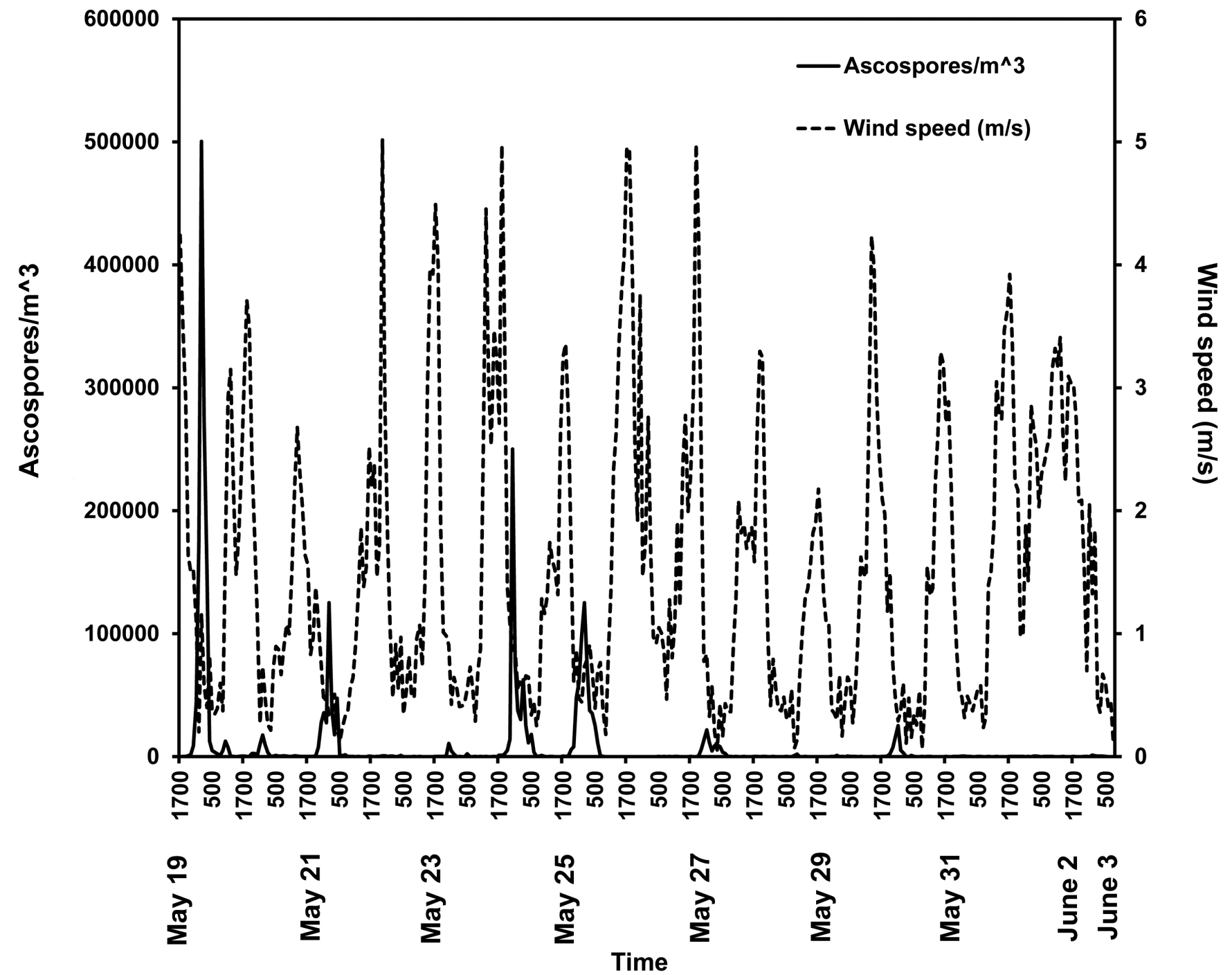

Fig. 4. Daily ascospore concentration for a field-scale source of Fusarium graminearum in 2011 (solid line). Ascospores were captured using a Quest volumetric spore sampler placed in the center of a 1-acre wheat field inoculated with FGVA4. In 2011, ascospores were continually sampled between 1700 hours 19 May and 0800 hours 3 June corresponding to when corn stalks inoculated with FGVA4 were present in the 1-acre wheat field. Major release events were observed when inoculum was present in the field. Daily patterns of wind speed are also given for reference (dashed line). 
perithecia dry out during the day when the humidity is low, but then are released during the evening when relative humidity increases $(21,36)$. Previous studies found that ascospore release is diminished during constant rainfall or high relative humidity $(11,21)$. In 2012, the time period that ascospore release was highest was between 2200 and 2300, while in 2011, between 0100 and 0200 showed the highest amount of ascospore release. Possible explanations for this small difference $(3 \mathrm{~h})$ in peak release interval include differences in environmental conditions and timing of the experiments (the samples were collected approximately 1 month earlier in 2012 than in 2011). We observed major release events; $41 \%(256,905 / 627,165)$ and $28 \%(80,190 / 288,508)$ of all of the ascospores captured by the volumetric spore sampler during the field sampling period were captured during single days in 2011 (20 May) and 2012 (9 May), respectively. This suggests that ascospores are not consistently released at the same rate each day and environmental conditions must be appropriate to trigger ascospore releases $(21,25,34-36)$. Understanding the timing of release of $F$. graminearum ascospores is important when trying to validate longdistance transport models, as one of the components of the models is spore release (1-4). Transport models must be calibrated to account for variability in release patterns of $F$. graminearum ascospores.

Estimates of ascospore production for our field-scale sources of inoculum were approximately 400 million ascospores/day over 10 days for both 2011 and 2012, when using variables (number of perithecia $/ \mathrm{cm}^{2}$ and number of ascospores discharged) collected from corn stalks. It is important to acknowledge that this is only an estimate, since field and laboratory observations may differ. One difference between laboratory and field conditions is relative hu-

Table 2. Daily ascospore production of the inoculated field using measurements collected from perithecia excised from a natural substrate $(\text { corn stalk })^{\mathrm{a}}$

\begin{tabular}{lc}
\hline $\begin{array}{l}\text { Days after perithecia } \\
\text { maturity }\end{array}$ & $\begin{array}{c}\text { Ascospore production } \\
\text { (ascospores released/day) }\end{array}$ \\
\hline 1 & $4.8 \times 10^{7}$ \\
2 & $3.3 \times 10^{8}$ \\
3 & $6.3 \times 10^{8}$ \\
4 & $1.0 \times 10^{9}$ \\
5 & $1.5 \times 10^{9}$ \\
6 & $1.9 \times 10^{8}$ \\
7 & $5.7 \times 10^{7}$ \\
8 & $1.3 \times 10^{7}$ \\
9 & $0.0 \times 10^{0}$ \\
10 & $0.0 \times 10^{0}$ \\
Mean $Q_{0}$ & $3.7 \times 10^{8}$ \\
\hline
\end{tabular}

a To calculate ascospore production, equation 1 was used and the number of ascospores released each day is shown in Figure 2. The estimates of ascospore production do not consider the fraction of spores that escape the crop canopy.

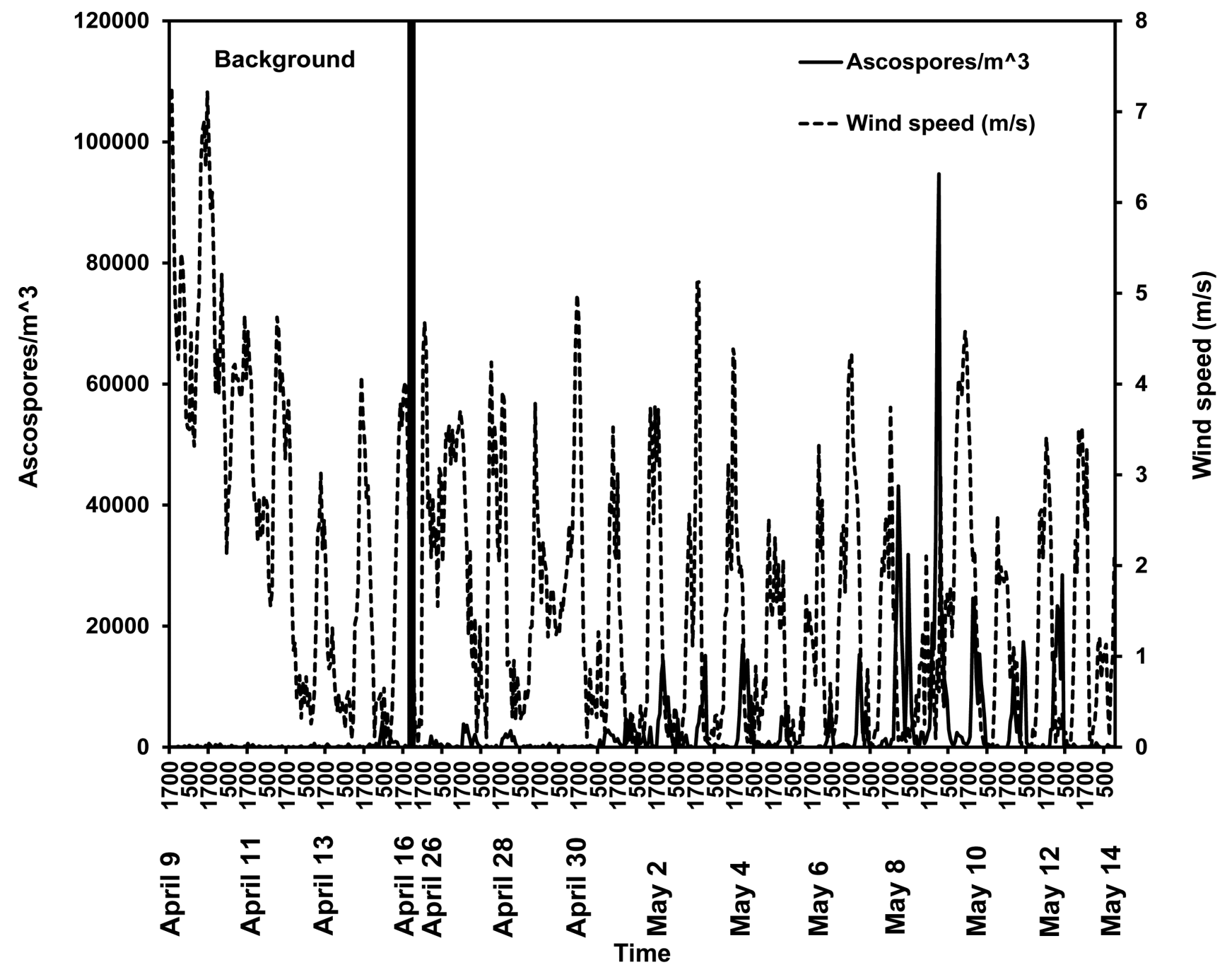

Fig. 5. Daily ascospore concentration for a field-scale source of Fusarium graminearum in 2012 (solid line). Ascospores were captured using a Quest volumetric spore sampler placed in the center of a 1-acre wheat field inoculated with FGVA4. Samples were collected from 1700 hours 9 April to 0400 hours 16 April corresponding to the week before corn stalks inoculated with FGVA4 were released in the 1-acre wheat field to assess the background source potential. After inoculated corn stalks were released in the 1-acre wheat field and mature perithecia formed, samples were collected with the volumetric spore sampler between 1800 hours 26 April and 1100 hours 14 May. Major release events were observed when inoculum was present in the field. Daily patterns of wind speed are also given for reference (dashed line). 
midity, which does not vary as much in the laboratory as it does in the field. Previous research has indicated that the change in relative humidity is an important factor in triggering ascospore release $(21,36)$. Additionally, it is important to address that under a laboratory setting we are assuming all the perithecia have formed and are ready to discharge their ascospores at the same time. However, in the field, perithecia formation and ascospore release may occur in "waves" as the conditions become favorable.

Spore production is one of the most difficult components to estimate in transport models for plant-pathogenic fungi (1-4). The work presented here provides an experimental means to estimate ascospore production of a 1-acre field-scale source of $F$. graminearum inoculum. Future work will examine how to adjust this estimate to account for variable weather conditions in the field leading to changes in spore production and release. The work presented here may be used to validate current spore transport models by combining the knowledge of spore production, spore release patterns, and field results for spore transport and deposition $(1-4,23)$. After validation of spore transport models, spore production estimates could be integrated into current FHB risk assessment tools, which currently only consider environmental factors for disease potential, to have a more complete risk assessment tool that takes into account both spore transport and environmental factors $(7,8)$.

\section{Acknowledgments}

This material is based upon work supported by the National Science Foundation under Grant Numbers DEB-0919088 (Atmospheric transport barriers and the biological invasion of toxigenic fungi in the genus Fusarium), CMMI1100263 (Dynamical mechanisms influencing the population structure of airborne pathogens: Theory and observations), DGE-0966125 (IGERT: MultiScale Transport in Environmental and Physiological Systems (MultiSTEPS)), and Virginia Small Grains Board proposals number 11-2660-06 and 12-2562-05 (Tracking the long-distance transport of the fungus that causes Fusarium head blight in wheat and barley). Any opinions, findings, and conclusions or recommendations expressed in this material are those of the authors and do not necessarily reflect the views of the National Science Foundation or the Virginia Small Grains Board.

\section{Literature Cited}

1. Aylor, D. E. 1986. A framework for examining inter-regional aerial transport of fungal spores. Agric. For. Meteorol. 38:263-288.

2. Aylor, D. E. 1999. Biophysical scaling and the passive dispersal of fungus spores: Relationship to integrated pest management strategies. Agric. For. Meteorol. 97:275-292.

3. Aylor, D. E., and Sutton, T. B. 1992. Release of Venturia inaequalis ascospores during unsteady rain: Relationship to spore transport and deposition. Phytopathology 82:532-540.

4. Aylor, D. E., Taylor, G. S., and Raynor, G. S. 1982. Long-range transport of tobacco blue mold spores. Agric. Meteorol. 27:217-232.

5. Barnett, H., and Lilly, V. G. 1947. The effects of biotin upon the formation and development of perithecia, asci and ascospores by Sordaria fimicola Ces. and de Not. Am. J. Bot. 34:196-204.

6. Beyer, M., Röding, S., Ludewig, A., and Verreet, J. A. 2004. Germination and survival of Fusarium graminearum macroconidia as affected by environmental factors. J. Phytopathol. 152:92-97.

7. De Wolf, E. D., Madden, L. V., and Lipps, P. E. 2003. Risk assessment models for Wheat Fusarium head blight epidemics based on within-season weather data. Phytopathology 93:428-435.

8. Del Ponte, E. M., Fernandes, J. M. C., Pavan, W., and Baethgen, W. E. 2009. A model-based assessment of the impacts of climate variability on Fusarium head blight seasonal risk in southern Brazil. J. Phytopathol. 157:675-681.

9. Doohan, F., Brennan, J., and Cooke, B. 2003. Influence of climatic factors on Fusarium species pathogenic to cereals. Eur. J. Plant Pathol. 109:755768.

10. Dufault, N., De Wolf, E., Lipps, P., and Madden, L. 2006. Role of temperature and moisture in the production and maturation of Gibberella zeae perithecia. Plant Dis. 90:637-644

11. Fernando, W. G., Miller, J. D., Seaman, W. L., Seifert, K., and Paulitz, T. C. 2000. Daily and seasonal dynamics of airborne spores of Fusarium graminearum and other Fusarium species sampled over wheat plots. Can. J. Bot. 78:497-505.

12. Hall, R. 1971. Effect of carbon-nitrogen ratios on production of perithecia by Sordaria fimicola. Can. J. Microbiol. 17:132-134.

13. Isard, S. A., Gage, S. H., Comtois, P., and Russo, J. M. 2005. Principles of the atmospheric pathway for invasive species applied to soybean rust. Bioscience 55:851-861.

14. Khonga, E., and Sutton, J. 1988. Inoculum production and survival of Gibberella zeae in maize and wheat residues. Can. J. Plant Pathol. 10:232239.

15. Klittich, C., and Leslie, J. 1988. Nitrate reduction mutants of Fusarium moniliforme (Gibberella fujikuroi). Genetics 118:417-423.

16. Kourik, R. 2005. Designing and maintaining your edible landscape naturally. Chelsea Green Publishing, White River Jct., VT.

17. Maldonado-Ramirez, S. L. 2001. Aerobiology of the wheat scab fungus, Gibberella zeae: Discharge, atmospheric dispersal, and deposition of ascospores. Ph.D. thesis. Cornell University, Ithaca, NY.

18. Maldonado-Ramirez, S. L., Schmale, D. G., III, Shields, E. J., and Bergstrom, G. C. 2005. The relative abundance of viable spores of Gibberella zeae in the planetary boundary layer suggests the role of longdistance transport in regional epidemics of Fusarium head blight. Agric. For. Meteorol. 132:20-27.

19. McMullen, M., Jones, R., and Gallenberg, D. 1997. Scab of wheat and barley: A re-emerging disease of devastating impact. Plant Dis. 81:13401348 .

20. Oke, T. R. 1987. Boundary Layer Climates. 2 ed. Cambridge University Press, Cambridge, UK.

21. Paulitz, T. C. 1996. Diurnal release of ascospores by Gibberella zeae in inoculated wheat plots. Plant Dis. 80:674-678.

22. Paulitz, T. C. 1999. Fusarium head blight: A re-emerging disease. Phytoprotection (Québec) 80:127-133.

23. Prussin, A. J., II, Li, Q., Malla, R., Ross, S. D., and Schmale, D. G., III. 2014. Monitoring the long distance transport of Fusarium graminearum from field-scale sources of inoculum. Plant Dis. 98:504-511.

24. Schmale, D., Ross, S., Fetters, T., Tallapragada, P., Wood-Jones, A., and Dingus, B. 2012. Isolates of Fusarium graminearum collected 40-320 meters above ground level cause Fusarium head blight in wheat and produce trichothecene mycotoxins. Aerobiologia 28:1-11.

25. Schmale, D. G., III, Arntsen, Q. A., and Bergstrom, G. C. 2005. The forcible discharge distance of ascospores of Gibberelia zeae. Can. J. Plant Pathol. 27:376-382.

26. Schmale, D. G., III, and Bergstrom, G. C. 2003. Fusarium head blight in wheat. The Plant Health Instructor DOI:10.1094/PHI-I-2003-0612-01.

27. Schmale, D. G., III, and Bergstrom, G. C. 2004. Spore deposition of the ear rot pathogen, Gibberella zeae, inside corn canopies. Can. J. Plant Pathol. 26:591-595.

28. Schmale, D. G., III, Bergstrom, G. C., and Shields, E. J. 2006. Night-time spore deposition of the Fusarium head blight pathogen, Gibberella zeae, in rotational wheat fields. Can. J. Plant Pathol. 28:100-108.

29. Schmale, D. G., III, Leslie, J. F., Zeller, K. A., Saleh, A. A., Shields, E. J., and Bergstrom, G. C. 2006. Genetic structure of atmospheric populations of Gibberella zeae. Phytopathology 96:1021-1026.

30. Shouru, S., Qiusheng, Y., Xiaoyu, D., and Jiqing, W. 2008. Analysis of mineral element contents and physical and chemical properties of corn stalk substrate. Trans. Chinese Soc. Agric. Eng. 24:41-44.

31. Snijders, C. H. A. 1990. Fusarium head blight and mycotoxin contamination of wheat, a review. Eur. J. Plant Pathol. 96:187-198.

32. Sutton, J. C. 1982. Epidemiology of wheat head blight and maize ear rot caused by Fusarium graminearum. Can. J. Plant Pathol. 4:195-209.

33. Trail, F., and Common, R. 2000. Perithecial development by Gibberella zeae: A light microscopy study. Mycologia 92:130-138.

34. Trail, F., Gaffoor, I., and Vogel, S. 2005. Ejection mechanics and trajectory of the ascospores of Gibberella zeae (anamorph Fuarium graminearum). Fungal Genet. Biol. 42:528-533.

35. Trail, F., Xu, H., Loranger, R., and Gadoury, D. 2002. Physiological and environmental aspects of ascospore discharge in Gibberella zeae (anamorph Fusarium graminearum). Mycologia 94:181-189.

36. Tschanz, A., Horst, K., and Nelson, P. E. 1975. Ecological aspects of ascospore discharge in Gibberella zeae. Phytopathology 65:597-599. 